\title{
Predicted Lifetime Health Outcomes for Aducanumab in Patients with Early Alzheimer's Disease
}

\author{
William L. Herring · Ian Gopal Gould · Howard Fillit • \\ Peter Lindgren · Fiona Forrestal · Robin Thompson · Peter Pemberton-Ross
}

Received: June 23, 2021 / Accepted: August 5, 2021 / Published online: August 23, 2021

(C) The Author(s) 2021

\begin{abstract}
Introduction: Alzheimer's disease $(\mathrm{AD})$ is a chronic and progressive neurodegenerative disease that places a substantial burden on patients and caregivers. Aducanumab is the first $\mathrm{AD}$ therapy approved by the US Food and Drug Administration to reduce a defining pathophysiological feature of the disease, brain amyloid plaques. In the phase 3 clinical trial
\end{abstract}

Supplementary Information The online version contains supplementary material available at https:// doi.org/10.1007/s40120-021-00273-0.

W. L. Herring · I. G. Gould

RTI Health Solutions, Research Triangle Park, NC, USA

H. Fillit

The Icahn School of Medicine at Mount Sinai, New York, NY, USA

H. Fillit

The Alzheimer's Drug Discovery Foundation, New

York, NY, USA

P. Lindgren

The Swedish Institute for Health Economics,

Stockholm, Sweden

F. Forrestal · R. Thompson

Biogen International GmbH, Baar, Switzerland

P. Pemberton-Ross $(\bowtie)$

Biogen International GmbH, Neuhofstrasse 30, 6340 Baar, Switzerland

e-mail: peter.pembertonross@biogen.com
EMERGE (NCT02484547), aducanumab reduced clinical decline in patients with mild cognitive impairment (MCI) due to $\mathrm{AD}$ and mild $\mathrm{AD}$ dementia and confirmed amyloid pathology.

Methods: We used a Markov modeling approach to predict the long-term clinical benefits of aducanumab for patients with early $\mathrm{AD}$ based on EMERGE efficacy data. In the model, patients could transition between $\mathrm{AD}$ severity levels (MCI due to $\mathrm{AD}$; mild, moderate, and severe $\mathrm{AD}$ dementia) and care settings (community vs. institution) or transition to death. The intervention was aducanumab added to standard of care (SOC), and the comparator was SOC alone. Data sources for base-case and scenario analyses included EMERGE, published National Alzheimer's Coordinating Center analyses, and other published literature.

Results: Per patient over a lifetime horizon, aducanumab treatment corresponded to 0.65 incremental patient quality-adjusted life-years (QALYs) and 0.09 fewer caregiver QALYs lost compared with patients treated with SOC. Aducanumab treatment translated to a lower lifetime probability of transitioning to $\mathrm{AD}$ dementia, a lower lifetime probability of transitioning to institutionalization $(25.2 \%$ vs. $29.4 \%$ ), delays in the median time to transition to AD dementia ( 7.50 vs. 4.92 years from MCI to moderate AD dementia or worse), and an incremental median time in the community of 1.32 years compared with SOC. 
Conclusion: The model predicted long-term benefits of aducanumab treatment in patients with MCI due to $\mathrm{AD}$ and mild $\mathrm{AD}$ dementia and their caregivers. The predicted outcomes provide a foundation for healthcare decision-makers and policymakers to understand the potential clinical and socioeconomic value of aducanumab.

Keywords: Aducanumab; Alzheimer's disease; Alzheimer's dementia; Amyloid plaques; Disease-modifying therapy; Institutionalization; Economic model; Markov model; Natural history; Progression

\section{Key Summary Points}

Alzheimer's disease (AD) is a chronic and progressive neurodegenerative disease that is estimated to cause $60-80 \%$ of all dementia cases.

Aducanumab, the first US Food and Drug Administration-approved $\mathrm{AD}$ treatment to reduce a defining pathophysiological feature of the disease, brain amyloid plaques, reduced clinical decline in patients with early AD in the phase 3 trial EMERGE.

Our objective was to predict the long-term clinical benefits of aducanumab for patients with early AD when the EMERGE efficacy data are extrapolated over patients' remaining lifetimes.

Aducanumab treatment was predicted to translate to a lower probability of patients transitioning to an AD dementia health state and an institutionalized care setting and delays in the median time to transition to $\mathrm{AD}$ dementia and institutionalization.

Predicted health outcomes provide a foundation for healthcare decisionmakers and policymakers to understand the potential clinical and socioeconomic value of aducanumab.

\section{INTRODUCTION}

Approximately 54 million people are living with dementia in the world today [1], with a projected rise to 152 million by 2050 [2]. Alzheimer's disease $(\mathrm{AD})$ is a chronic and progressive neurodegenerative disease that is estimated to cause $60-80 \%$ of all dementia cases [3]. Clinical $\mathrm{AD}$ is associated with shortened life expectancy [3], and represents the fifth leading cause of death in the United States (US) in those older than 65 years of age [4]. The pathological hallmarks of $\mathrm{AD}$ include extracellular amyloid beta (A $\beta)$ deposition, intracellular pathologic tau, and neurodegeneration [5]; some of these pathophysiological changes precede the onset of clinical symptoms by up to 20 years [6-9]. AD lies on a continuum, which progresses from mild cognitive impairment (MCI) to mild, moderate, and severe stages of dementia $[3,9]$.

In the US, an estimated 9-10 million people aged 50 years or older have MCI that would clinically be attributed to AD; this corresponds to approximately 1 in 12 people who are 50 years of age or older, considering the increasing prevalence of MCI with age and excluding the approximately $25 \%$ of MCI cases that are clinically determined to result from causes other than AD [10-13]. Further applying data on $\mathrm{AD}$ biomarker status, approximately 1 in 25 individuals (4\%) aged 50 years or older are estimated to have MCI with sufficient amyloid load for amyloid positivity to be confirmed using biomarker tests [14].

Individuals with $\mathrm{MCI}$ due to $\mathrm{AD}$ can still perform adequately and independently in daily life activities; however, as AD severity increases, neurobehavioral symptoms and cognitive function worsen [9]. As patients transition from mild to moderate $\mathrm{AD}$ dementia, they require increasing levels of assistance with daily life activities. In earlier stage $\mathrm{AD}$, they begin to require occasional assistance with complex activities, later progressing to needing frequent help with basic needs [9]. Patients with severe $\mathrm{AD}$ dementia require full-time care with the most basic feeding, toileting, and ambulating needs [9]. The progressive reduction in both independence and quality of life that patients 
with $\mathrm{AD}$ experience renders caregivers at increased risk of emotional distress, negative mental and physical well-being, reduced workplace productivity, and loss of earnings $[3,15,16]$.

Beyond the emotional toll, $\mathrm{AD}$ imposes a significant financial burden for people living with the disease and their caregivers [4, 15-18]. Even though direct medical costs paid by the healthcare system may represent only $16 \%$ of total AD costs [19], the substantial socioeconomic costs resulting from $\mathrm{AD}$ are not traditionally included in health technology assessments [17, 20-22]. Patients with AD eventually require multidisciplinary care from family and friends, caregiving professionals, and the medical community [17]. Approximately $80 \%$ of patients with $\mathrm{AD}$ and related dementias in the US live at home, with family and community caregivers contributing an estimated 18.5 billion hours of unpaid care in 2019 [4].

Pharmacological therapies are available to address the symptoms associated with the disease, and are considered standard of care (SOC) for $\mathrm{AD}$ dementia management; these symptomatic therapies are not approved for patients with MCI due to AD [23]. Symptomatic therapies approved in the US include the cholinesterase inhibitors donepezil, rivastigmine, and galantamine in addition to memantine, an $\mathrm{N}$ methyl-D-aspartate receptor antagonist [24]. A combination therapy, memantine and donepezil, is also approved in the US [24].

Aducanumab is the first $\mathrm{AD}$ therapy approved by the US Food and Drug Administration (FDA) to reduce a defining pathophysiological feature of the disease: brain amyloid beta plaques [25]. Aducanumab is a human, immunoglobulin gamma 1 (IgG1) monoclonal antibody directed against aggregated soluble and insoluble forms of $A \beta$ [26], a defining pathophysiological feature of $\mathrm{AD}$ [25]. Aducanumab was approved under accelerated approval based on reduction in $A \beta$ plaques observed in patients treated with aducanumab; continued approval for this indication may be contingent upon verification of clinical benefit in confirmatory trial(s) [25].
The first predictive computer-based disease models for $\mathrm{AD}$ were developed to evaluate symptomatic therapies, whereas subsequent models have been used to predict the long-term benefits of hypothetical treatments that modify the course of $\mathrm{AD}$ [21]. These models, some of which include the MCI due to AD stage of the disease, reflect a broad range of approaches (e.g., patient-level simulation, simple and multidimensional Markov model structures) that rely on diverse data sources. Many of these models of $\mathrm{AD}$ rely on data from only one symptom domain and rarely account for the critical role of biomarkers to accurately stage $\mathrm{AD}$ or the impact of biomarkers on AD-related health outcomes [21]. A breakthrough treatment that delays the onset or slows the progression of $\mathrm{AD}$ is likely to be more successful if diagnosis occurs at the early AD stage to reduce the probability of patients transitioning to a more severe $\mathrm{AD}$ health state. Such a therapy would reduce indirect and social care costs and delay direct medical costs [21], and may necessitate a revised framework for value assessments in AD [22].

The identically designed, randomized, double-blind, placebo-controlled, global phase 3 trials EMERGE (NCT02484547) and ENGAGE (NCT02477800) assessed the efficacy and safety of low-dose and high-dose aducanumab in patients with $\mathrm{MCI}$ due to $\mathrm{AD}$ and mild $\mathrm{AD}$ dementia, and confirmed amyloid pathology [25]. The primary endpoint was a change from baseline in the Clinical Dementia Rating Sum of Boxes (CDR-SB) at week 78. Patients received an intravenous dose of aducanumab every 4 weeks in addition to SOC; participants receiving placebo were managed by SOC only, which permitted a stable, concomitant use of symptomatic therapies that were prescribed prior to baseline at the discretion of physicians. The EMERGE and ENGAGE trials were halted based on an interim futility analysis of the data pooled from both studies. Subsequent efficacy analyses from a larger dataset, collected up to futility declaration, and that followed prespecified statistical analyses, showed that the primary, secondary, and tertiary efficacy endpoints were met in the high-dose group of EMERGE, though not in ENGAGE [25]. 
Our objective was to develop and use an evidence-based modeling approach to predict potential long-term health outcomes, including delays for transitions to more advanced $\mathrm{AD}$ severity health states and institutionalization, and survival in the community (as opposed to institutionalization) in aducanumab-treated populations. Using high-dose aducanumab data from EMERGE, we present the first model to include efficacy data from a randomized controlled trial of an agent that reduces $A \beta$ plaques in a population of $\mathrm{MCI}$ due to $\mathrm{AD}$ and mild $\mathrm{AD}$ dementia with confirmed amyloid pathology. Data from ENGAGE were not used in the current model because fewer patients in the ENGAGE trial had sustained and consistent dosing at $10 \mathrm{mg} / \mathrm{kg}$ dose compared with those in EMERGE. The decision to choose the trial with sufficient exposure to high-dose treatment was made in light of the importance of the strong association between treatment exposure and clinical outcomes [25].

\section{METHODS}

\section{Modeling Approach}

A Markov-based cohort model with health states for $\mathrm{AD}$ severity levels, care settings, and death was programmed in Microsoft Excel (Microsoft, Redmond, WA, USA). The AD severity levels used in the model were MCI due to $\mathrm{AD}$, mild $\mathrm{AD}$ dementia, moderate $\mathrm{AD}$ dementia, and severe AD dementia. At each severity level, patients could be cared for in community or institutional settings. The model structure aligns with previously published Markov models for AD in which MCI due to AD was included as a health state [27-30]. Health state definitions for the model were defined by CDR-SB ranges [31] to align with the efficacy analyses for aducanumab from the EMERGE clinical trial. The CDR-SB ranges were as follows: MCI due to $\mathrm{AD}$ (0.5-4.0), mild AD (4.5-9.0), moderate $\mathrm{AD}$ (9.5-15.5), and severe AD (16.0-18.0) [31]. Because some of the data used in the model referenced severity levels defined by global CDR or Mini-Mental State Examination (MMSE) scores, a crosswalk of $\mathrm{AD}$ severity definitions considered in this study is shown in Table S1 in the electronic supplementary material.

To accommodate the diversity of available sources with $\mathrm{AD}$ progression data, the model was designed to simulate transitions between any pair of non-death health states, as shown in a simplified schematic in Fig. 1. In addition to transitions to the next severity level (e.g., MCI due to $\mathrm{AD}$ to mild $\mathrm{AD}$ dementia), the model allowed transitions between non-sequential severity levels (e.g., from MCI due to AD to moderate $\mathrm{AD}$ dementia) and to less severe health states (e.g., from moderate AD dementia to mild $\mathrm{AD}$ dementia). Health states at each severity level of AD dementia were considered separately for individuals in community versus institutionalized (i.e., long-term care facilities) settings. Transitions from community to institutionalization and vice versa were permitted in accordance with available data sources. The model permitted transitions to death from any health state. In alignment with previously published Markov models for $\mathrm{AD}$, transitions were modeled on an annual cycle, and a halfcycle correction was applied to model outcomes in accordance with best practices for statetransition modeling [32].

For the model, the intervention was highdose aducanumab in addition to SOC (Adu + SOC), and the comparator was SOC alone. Non-pharmacological interventions, such as care delivery coordination, nutrition programs, and exercise, were considered to be reflected in the outcomes (progression, utilities) for the SOC.

\section{Model Parameters}

The EMERGE trial was conducted in accordance with Declaration of Helsinki and the International Conference on Harmonization and Good Clinical Practice guidelines and was approved by ethics committees or institutional review boards at each site. All patients provided written informed consent. An independent data monitoring committee was responsible for monitoring the conduct of the trials, assessing safety routinely, and reviewing futility analysis results. 


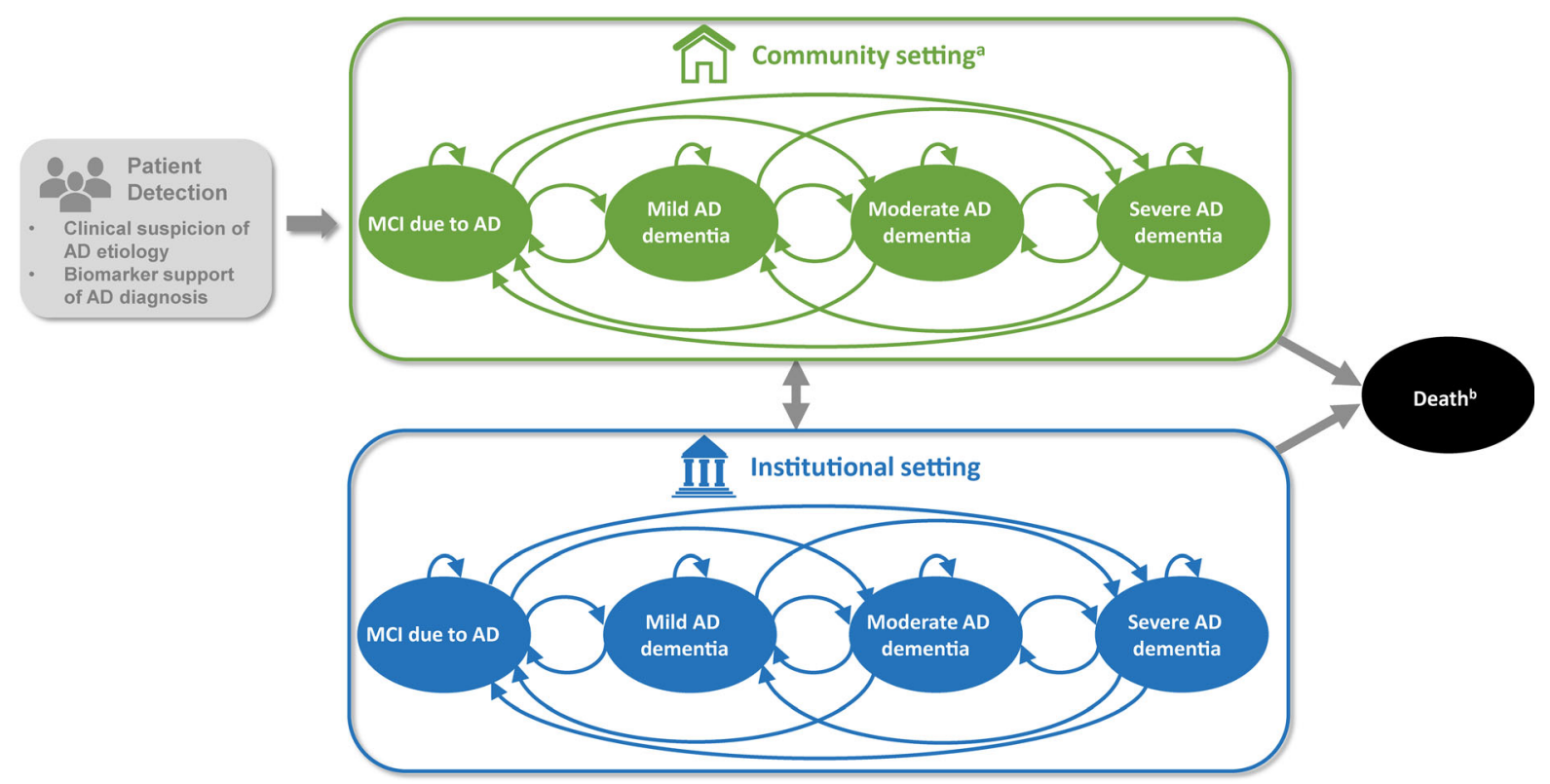

Fig. 1 Markov-based model. ${ }^{a}$ Not all transitions shown in the model structure were included in the base-case analysis. The included transitions were based on the selected natural history data. Transitions between $\mathrm{AD}$ severity levels were

This article is based on previously conducted studies and does not contain any new studies with human participants or animals performed by any of the authors.

\section{Baseline Population Characteristics}

The baseline population characteristics for the model were established using pooled data from the high-dose aducanumab and placebo arms of the EMERGE trial [25]. The EMERGE trial included patients with MCI due to $\mathrm{AD}$ [33] and mild AD dementia [34] at baseline. The mean age of the participants was 70.7 years (Table 1). It is anticipated that patients in real-world settings would receive a diagnosis of $\mathrm{AD}$ and initiate treatment at a younger age than in contemporary clinical trial settings; therefore, the baseline cohort used in the base-case analysis was designated as individuals aged 65.0 years with MCI due to AD (Table 1). Alternative populations defined by age and baseline health-state distributions (e.g., a cohort of individuals with a baseline mix of MCI due to assumed to be independent of the care setting (i.e., community vs. institutional). ${ }^{\mathrm{b}}$ Transitions to death could occur from all other health states. $A D$ Alzheimer's disease, $M C I$ mild cognitive impairment

$\mathrm{AD}$ and mild $\mathrm{AD}$ dementia) were considered in scenario analyses.

\section{Natural History}

Disease Progression Model parameters for the natural history progression of $\mathrm{AD}$ reflected the expected experiences of patients managed according to the SOC. Where available, datasets with sufficient numbers of biomarker-positive individuals were chosen to more closely align with the EMERGE trial design and aducanumab eligibility criteria. The National Alzheimer's Coordinating Center (NACC) Uniform Data Set analysis by Potashman et al. [35] was selected as the primary source to model disease progression in the base case, because the analysis included an amyloid-confirmed population and used CDR-SB to define health states. Using the NACC data, Potashman et al. [35] calculated transition probabilities in "incident" patients for whom entry into a new AD health state was observed in the data, thereby increasing the reliability of the transition probability estimates by capturing the full time spent in a health state. 
Table 1 Baseline population characteristics and natural history parameters

\begin{tabular}{|c|c|c|}
\hline Baseline population characteristics & Values & Source \\
\hline \multicolumn{3}{|l|}{$\mathrm{MCl}$ due to $\mathrm{AD}$ only cohort (base case) } \\
\hline Age, years & 65.0 & \multirow{2}{*}{$\begin{array}{l}\text { Assumption (age and health } \\
\text { state); EMERGE (female) }\end{array}$} \\
\hline Female, \% & 52.4 & \\
\hline \multicolumn{3}{|l|}{ Health-state distribution, \% } \\
\hline $\mathrm{MCl}$ due to $\mathrm{AD}$ & 100.00 & \multirow{7}{*}{ EMERGE } \\
\hline Mild AD dementia & 0.00 & \\
\hline \multicolumn{3}{|c|}{$\begin{array}{l}\mathrm{MCl} \text { due to } \mathrm{AD} \text { and mild } \mathrm{AD} \text { dementia cohort } \\
\text { (scenario) }\end{array}$} \\
\hline Age, years & 70.7 & \\
\hline Female, \% & 52.4 & \\
\hline \multicolumn{2}{|l|}{ Health-state distribution, \% } & \\
\hline $\mathrm{MCl}$ due to $\mathrm{AD}$ & 80.7 & \\
\hline Mild AD dementia & 19.3 & \\
\hline \multicolumn{3}{|l|}{ Annual transition probabilities } \\
\hline \multicolumn{3}{|l|}{ From $\mathrm{MCl}$ due to $\mathrm{AD}$ to $\mathrm{AD}$ dementia } \\
\hline Annual probability, $\%$ & 23.2 & \multirow[t]{5}{*}{ Potashman et al. 2020 (35) } \\
\hline \multicolumn{2}{|c|}{ Landing spot distribution in AD dementia, \% } & \\
\hline Mild AD dementia & 72.7 & \\
\hline Moderate AD dementia & 27.3 & \\
\hline Severe AD dementia & 0.0 & \\
\hline \multicolumn{3}{|l|}{ From mild AD dementia to, $\%$} \\
\hline $\mathrm{MCl}$ due to $\mathrm{AD}$ & 3.3 & \multirow[t]{3}{*}{ Potashman et al. 2020 (35) } \\
\hline Moderate AD dementia & 35.2 & \\
\hline Severe AD dementia & 4.4 & \\
\hline \multicolumn{3}{|l|}{ From moderate AD dementia to, $\%$} \\
\hline $\mathrm{MCl}$ due to $\mathrm{AD}$ & 0.0 & \multirow[t]{3}{*}{ Potashman et al. 2020 (35) } \\
\hline Mild AD dementia & 2.9 & \\
\hline Severe AD dementia & 42.0 & \\
\hline \multicolumn{3}{|l|}{ From severe $A D$ dementia to, $\%$} \\
\hline $\mathrm{MCl}$ due to $\mathrm{AD}$ & 0.0 & \multirow{3}{*}{ Potashman et al. 2020 (35) } \\
\hline Mild AD dementia & 0.0 & \\
\hline Moderate AD dementia & 1.9 & \\
\hline \multicolumn{3}{|l|}{ Institutionalization } \\
\hline \multicolumn{3}{|l|}{ Proportion institutionalized, $\%$} \\
\hline $\mathrm{MCl}$ due to $\mathrm{AD}$ & 1.7 & \multirow{4}{*}{ Davis et al. 2018 (36) } \\
\hline Mild AD dementia & 4.3 & \\
\hline Moderate AD dementia & 11.6 & \\
\hline Severe AD dementia & 43.2 & \\
\hline \multicolumn{3}{|l|}{ Mortality } \\
\hline \multicolumn{3}{|c|}{ HRs for mortality (vs. general population) } \\
\hline $\mathrm{MCl}$ due to $\mathrm{AD}$ & 1.48 & \multirow{4}{*}{ Wilson et al. 2009 (41) } \\
\hline Mild AD dementia & 2.84 & \\
\hline Moderate AD dementia & 2.84 & \\
\hline Severe AD dementia & 2.84 & \\
\hline
\end{tabular}

$A D$ Alzheimer's disease, $H R$ hazard ratio, $M C I$ mild cognitive impairment

Institutionalization and Number of Caregivers The latest available institutionalization estimates leveraging NACC data and including patients with MCI due to $\mathrm{AD}$ [36] were used to model institutionalization; many of the considered publications reporting institutionalization rates did not include a distinct MCI population [37-39]. In the base case, prevalence-based institutionalization data were used that reflect the proportion of patients institutionalized at each AD severity level [36] (Table 1). In a scenario analysis, incidence-based institutionalization 
data were used from a study reporting annual transition probabilities from the community to institutionalization by AD severity level [40]. Patients with $\mathrm{AD}$ commonly require more than one caregiver [15]; however, an assumption of one caregiver per patient throughout the $\mathrm{AD}$ continuum was used in the current model to align with available data on patient-caregiver dyads [15].

Mortality The base-case analysis used hazard ratio (HR) estimates reported by Wilson et al. [41] to model excess mortality in patients with AD (Table 1). The multiplicative approach suggested by Wilson et al. [41], i.e., HRs applied to age-specific general mortality estimates from Arias and $\mathrm{Xu}$ [42], was preferred for the model, because this approach naturally increases the probability of death across all health states as patients age. Notably, the Wilson et al. [41] study did not differentiate between AD dementia severity levels; therefore, the increased mortality risk associated with $\mathrm{AD}$ dementia was assumed to be the same across $\mathrm{AD}$ dementia severity levels. This study was selected for the base case because it included a distinct MCI population. Other potential data sources $[30,43]$ include a very mild AD dementia category, but it is unclear how comparable this is to MCI due to AD. In a scenario analysis, the HRs from Wimo et al. [30] were considered.

\section{Aducanumab Attributes}

\section{Dosing}

Patients in the aducanumab arms of EMERGE received an intravenous dose of either low-dose (3-6 mg/kg) or high-dose $(6-10 \mathrm{mg} / \mathrm{kg})$ aducanumab every 4 weeks, up to a maximum of 20 doses. Per the original EMERGE trial protocol (protocol version 1), the target dose in the highdose aducanumab group initially differed according to the apolipoprotein (ApoE) $\varepsilon 4$ carrier status of the patients: $6 \mathrm{mg} / \mathrm{kg}$ for carriers (titrated from 1 to 3 to $6 \mathrm{mg} / \mathrm{kg}$ over 24 weeks) and $10 \mathrm{mg} / \mathrm{kg}$ (titrated from 1 to 3 to 6 to $10 \mathrm{mg} / \mathrm{kg}$ over 24 weeks) for non-carriers. Based on the results of the phase $1 \mathrm{~b}$ PRIME clinical trial of aducanumab (NCT01677572) [26], two protocol amendments were implemented so that more patients could achieve the target dose of $10 \mathrm{mg} / \mathrm{kg}$. An amendment in protocol version 3 enabled patients who had suspended aducanumab dosing due to amyloid-related imaging abnormalities (ARIA) to resume dosing after resolution of the ARIA event. Dosing was resumed at the same dose prior to suspension and titration to the target dose continued. Because of the protocol version 3 amendment, ApoE $\varepsilon 4$ non-carriers who had an ARIA event had the opportunity to receive more $10 \mathrm{mg} / \mathrm{kg}$ target doses. An amendment in protocol version 4 enabled all patients in the high-dose group to receive $10 \mathrm{mg} / \mathrm{kg}$ aducanumab regardless of ApoE $\varepsilon 4$ carrier status [25].

\section{Treatment Effect}

Treatment effect estimates for aducanumab were based on a comparison between high-dose aducanumab (6 or $10 \mathrm{mg} / \mathrm{kg}$ ) and placebo in the EMERGE trial using the intention-to-treat (ITT) population as the base case. Although combining data from EMERGE and ENGAGE trials could add to the statistical power of the analysis, a compromise to focus only on the EMERGE trial was scientifically justified given the heterogeneity in exposure to aducanumab across the two studies for the ITT population. The ITT population in the EMERGE trial (placebo, $n=548$; high-dose aducanumab, $n=547$ ) was defined as all randomized patients who received at least one dose of study treatment. An alternative opportunity-to-complete (OTC) population was designated to replicate treatment effect estimates for aducanumab in scenario analyses. The OTC population was defined as all patients who had an opportunity to complete week 78 of the trial prior to March 20, 2019, when the trial was prematurely terminated.

Aducanumab demonstrated statistical significance on the prespecified primary and secondary clinical endpoints in the EMERGE trial. The primary endpoint was change from baseline in CDR-SB at week 78. The difference between high-dose aducanumab (1.35) versus placebo (1.74) was $-0.39(p=0.01)$, representing a $22 \%$ reduction in clinical decline in favor of aducanumab [25]. However, the current model 
requires the treatment effect of aducanumab to be expressed as a reduction in transition probabilities between discrete health states, defined using CDR-SB thresholds (Table S1 in the electronic supplementary material). This analysis was not predefined in the EMERGE Statistical Analysis Plan. A post hoc time-to-event analysis was conducted on the transition from MCI due to $\mathrm{AD}$ to $\mathrm{AD}$ dementia in the EMERGE trial. Only patients with MCI due to AD and mild AD dementia were enrolled in the EMERGE trial. Transition probabilities from mild AD dementia to more severe health states were not calculated due to the small sample size.

The typical approach to testing the equality of two survival curves is by using the log-rank test statistic. The log-rank test has maximum power under the proportional hazards assumption. When the hazard functions are not proportional, the power of the log-rank test is reduced, and the HR from Cox regression may be difficult to interpret. One type of nonproportionality is delayed clinical effect, in which the treatment does not immediately show an advantage, and a lag time is observed prior to the hazard functions diverging later in the follow-up period. This is expected based on the mechanism of action of aducanumab and the titration period. Alternative approaches to survival analysis are required when the assumption of proportional hazards does not hold, such as the Fleming-Harrington weighted log-rank statistic, defined as: $\mathrm{FH}(p, q)=\left[\widehat{S}\left(t_{j-1}\right)\right]^{p}\left[1-\widehat{S}\left(t_{j-1}\right)\right]^{q}$, $p \geq 0, q \geq 0$. When a nonproportional hazards pattern can be determined a priori, weighted logrank tests can be used to test early or late differences. However, these analyses were performed post hoc; therefore, it was not possible to predefine the weights, and all $p$ values presented were considered nominal. The $p$ value from the log-rank test was 0.0150 . When performing the Fleming-Harrington weighted log-rank test to test late differences ( $p=0, q=1$; i.e., giving more weight to later timepoints), the $p$ value was 0.0008 . Both tests show a nominally statistically significant treatment effect. However, considering the mechanism of action of aducanumab and the titration period, the weighted log-rank test to test late differences was the most appropriate.
Because CDR-SB was only assessed postbaseline at three timepoints, a piecewise proportional hazards model was not viable because one section would be based on a single timepoint. Therefore, the HR from the overall Cox model was presented. However, because the HR over the first 26 weeks appeared to be close to 1 , this increased the overall HR and therefore was considered a conservative estimate for later timepoints.

The Cox model estimated an HR of 0.690 (95\% CI 0.552-0.863) for aducanumab relative to placebo in the base-case ITT population. In the current model, this HR was assumed to equate to a $31.0 \%$ reduction in the annual probability of progression from MCI due to $\mathrm{AD}$ to AD dementia for patients treated with aducanumab. An assumption of a 31.0\% reduction in annual transition probability was also applied to patients with mild $\mathrm{AD}$ treated with aducanumab, because the EMERGE trial contained an insufficient number of patients to perform a separate survival analysis for this group. For the OTC population, the HR was $0.646 \quad(95 \%$ CI $0.495-0.844)$, which was assumed to equate to a $35.4 \%$ reduction in progression for patients treated with aducanumab.

\section{Duration of Effect and Discontinuation}

The long-term effect of aducanumab treatment in the model was informed by EMERGE data, including treatment efficacy, the durability of the treatment effect over time, clinical stopping rules, and treatment discontinuation. For the model, an assumption was made that the treatment effect of aducanumab derived from the EMERGE trial would last as long as patients remained on the treatment. This assumption is supported by the PRIME clinical trial, in which a clinical benefit of aducanumab was observed over 48 months of treatment [44].

In the model, treatment with aducanumab was stopped upon transition to moderate $\mathrm{AD}$ dementia or worse with no maximum time on treatment imposed; if patients subsequently transitioned to a less severe health state after stopping treatment due to progression (e.g., moderate $\mathrm{AD}$ dementia back to mild $\mathrm{AD}$ 
dementia), aducanumab treatment was not restarted.

In the base-case analysis, a discontinuation rate of $0.0 \%$ was assumed to reflect the experience of patients who were stable on treatment with aducanumab. Of the participants in the EMERGE trial treated with high-dose aducanumab, $11.0 \%$ discontinued treatment by the end of the 78-week trial period for reasons considered related to treatment (i.e., AEs, consent withdrawn, and other reasons). This translated to the $7.5 \%$ annual discontinuation rate used in the scenario analyses. Study-related reasons for discontinuation including the early termination of the study, study visit burden, and investigator decision were not included. Discontinuation rates in the model did not include temporary aducanumab dosing delays or interruptions (e.g., due to ARIA events).

\section{Adverse Events}

Adverse events (AEs) were eligible to be included in the model if they occurred in $\geq 10 \%$ of patients in either the high-dose aducanumab or placebo arm of EMERGE, or were at least two percentage points more common for participants treated with high-dose aducanumab than for those who received placebo. AEs that met the inclusion criteria for the model were fall, headache, nasopharyngitis, dizziness, diarrhea, and ARIA events, including superficial siderosis. The model did not differentiate between ARIA-E (ARIA-edema) and ARIA-H (ARIA-H microhemorrhage, ARIA-H superficial siderosis, or ARIA-H macrohemorrhage); while ARIA-E, ARIA-H microhemorrhage, and ARIA-H superficial siderosis met the eligibility criteria, they were only included in the model under the overall term of ARIA. In EMERGE, ARIA events were predominantly asymptomatic $(75.0 \%$ of incremental ARIA events) and resolved within months; aducanumab treatment was permanently discontinued in cases of severe ARIA [25]. Using the 78-week EMERGE trial safety data, incidence rates for all AEs, except ARIA events, were annualized and incorporated in the model for each year of treatment with aducanumab (Table S2 in the electronic supplementary material). ARIA incidence was not annualized, because ARIA events were assumed to occur only once, and were assigned to the first year of treatment with aducanumab only. Incremental $\mathrm{AE}$ rates for aducanumab were included in the analysis, because AEs that occurred in the placebo arm are reflected in AD management with SOC. This resulted in the exclusion of nasopharyngitis, because it was more common for patients in EMERGE treated with placebo than with high-dose aducanumab.

\section{Utilities}

Patient utilities for individuals with MCI due to $\mathrm{AD}$ and $\mathrm{AD}$ dementia were selected from a systematic literature review and meta-analysis by Landeiro et al. [45], which included selfreported and proxy-reported utilities for individuals at each $\mathrm{AD}$ severity health state (Table S3 in the electronic supplementary material). In the model, the proxy-reported European Quality of Life-5 Dimensions (EQ-5D) estimates were used because patients in later stages of AD dementia may provide less reliable self-rated assessments [46]. Landeiro et al. [45] included studies of patients in both community and institutionalized care settings, but the meta-analysis did not analyze the care settings separately. Therefore, for each level of $A D$ severity, the same utility values were used for patients living in the community or institutionalized settings, although it is known from the base-case data source for institutionalization used in the model that more patients are institutionalized as AD severity progresses [36].

Caregiver disutility values (Table S3 in the electronic supplementary material) were used in the model to account for the effects of $\mathrm{AD}$ on caregiver quality of life. Caregiver disutility values were estimated by subtracting EQ-5Drated caregiver utility values published by Mesterton et al. [47] from general population, age-adjusted utility values published by Janssen and Szende [48]. Because the study by Mesterton et al. [47] did not include caregivers of patients with $\mathrm{MCI}$ due to $\mathrm{AD}$, the caregiver disutilities for patients with MCI due to AD were assumed to be zero. While there is evidence that caregiver burden, as measured by hours of caregiving, persists after patients are 
institutionalized $[15,18]$, data on utility values for caregivers of institutionalized patients are limited. As such, we assumed caregiver disutilities were the same across both patient care settings.

Utility decrements were combined with the duration of AE impact to estimate the qualityadjusted life-years (QALYs) lost for each AE per year of aducanumab treatment, with separate values for year 1 (reflecting ARIA events and chronic AEs) and years 2 and beyond (reflecting chronic AEs only). Values for years 2 and beyond were applied for as long as patients continued treatment with aducanumab.

\section{Model Outcomes}

The model was used to estimate potential health outcomes for patients with $\mathrm{AD}$ and the impact on quality of life for patients and their caregivers. The base-case analysis was conducted over the patients' remaining lifetimes, with shorter horizons considered in scenario analyses. In addition to estimating outcomes separately for Adu + SOC and SOC, the model also estimated incremental outcomes. Eventbased health outcomes (undiscounted) included estimations of the proportions of patients progressing to more advanced health states (e.g., moderate $\mathrm{AD}$ dementia or worse), the median time to progression to these health states (the time when $50 \%$ of those alive had progressed), and the median time to institutionalization. Time-based health outcomes included estimations of total life-years (LYs) of survival (undiscounted) by AD severity level in both the community and institution settings. Quality-adjusted survival outcome estimates (discounted) included patient QALYs and caregiver QALYs lost. In alignment with Institute for Clinical and Economic Review recommendations [49], the equal value of LYs gained was estimated by applying age-specific general population utility values [48] to time periods corresponding to survival gains for aducanumab. For clinical outcomes that were discounted, an annual rate of $3.0 \%$ was used $[49,50]$.

\section{Model Validation}

The model was validated by comparing the predicted outcomes for the SOC with outcomes reported in recently published models for MCI due to $\mathrm{AD}$. The published models included in the comparison were selected to reflect the diversity of modeling approaches and data sources used to model the AD continuum. In particular, the selected models included a Markov model with a structure similar to that of the current model using Swedish Dementia (SveDem) registry data [30], an open-source Markov model (v.1.1) from the International PharmacoEconomic Collaboration on Alzheimer's Disease (IPECAD) with multi-dimensional symptom health states for dementia using NACC data [51, 52], a multi-state statistical model using combined data from six longitudinal cohort studies [53], and the Alzheimer's Disease-Archimedes Condition Event (AD-ACE) simulation model with continuous biomarker and symptom trajectories using data from the Alzheimer's Disease Neuroimaging Initiative and the Assessment of Health Economics in Alzheimer's Disease project [54]. The model comparisons were adjusted to account for baseline age. Other adjustments to model settings or data sources were not considered. The model comparison details are presented in the electronic supplementary materials, and the results are discussed below.

\section{RESULTS}

\section{Base-Case Analysis}

The extrapolation of the clinical benefits of aducanumab treatment from EMERGE were predicted across the patients' remaining lifetimes (Table 2) and over intermediate 5- and 10-year horizons (Table S4 in the electronic supplementary material).

Over a lifetime horizon, patients treated with aducanumab were estimated to have a lower lifetime probability of transitioning to $\mathrm{AD}$ dementia (i.e., mild, moderate, or severe $\mathrm{AD}$ dementia) compared with patients treated with SOC (Table 2; Fig. 2a). The estimated number of 
Table 2 Base-case model outcomes over lifetime horizon

\begin{tabular}{|c|c|c|c|}
\hline Event-based model outcomes (undiscounted) & soc & Adu + SOC & Incremental \\
\hline \multicolumn{4}{|l|}{ Number of transitions (net of return transitions), $\%$} \\
\hline To AD dementia (i.e. mild AD dementia or worse) & 89.4 & 82.8 & -6.5 \\
\hline To moderate AD dementia or worse & 80.0 & 69.5 & -10.6 \\
\hline To severe AD dementia & 67.2 & 57.1 & -10.1 \\
\hline To institutionalization & 29.4 & 25.2 & -4.1 \\
\hline \multicolumn{4}{|l|}{$\begin{array}{l}\text { Progression to AD dementia (i.e. mild AD dementia or worse), } \\
\text { years }\end{array}$} \\
\hline Median survival in $\mathrm{MCl}$ due to $\mathrm{AD}$ (of baseline cohort) & 2.54 & 3.70 & 1.16 \\
\hline Median time to AD dementia (of those alive) & 2.77 & 4.30 & 1.53 \\
\hline \multicolumn{4}{|l|}{ Progression to moderate AD dementia or worse, years } \\
\hline Median survival in mild AD dementia or better (of baseline cohort) & 4.29 & 5.95 & 1.66 \\
\hline Median time to moderate $\mathrm{AD}$ dementia (of those alive) & 4.92 & 7.50 & 2.58 \\
\hline \multicolumn{4}{|l|}{ Progression to severe AD dementia, years } \\
\hline $\begin{array}{l}\text { Median survival in moderate AD dementia or better (of baseline } \\
\text { cohort) }\end{array}$ & 6.12 & 7.63 & 1.51 \\
\hline Median time to severe AD dementia (of those alive) & 7.44 & 10.26 & 2.82 \\
\hline \multicolumn{4}{|l|}{ Time to institutionalization, years } \\
\hline Median survival in community (of baseline cohort) & 8.85 & 10.17 & 1.32 \\
\hline Median time to institutionalization (of those alive) & NR & NR & NA \\
\hline Time-based model outcomes & soc & Adu + SOC & Incremental \\
\hline Total LYs (survival; undiscounted) & 12.92 & 13.31 & 0.38 \\
\hline Years on treatment & NA & 7.03 & NA \\
\hline Community, total & 10.06 & 10.95 & 0.89 \\
\hline $\mathrm{MCl}$ due to $\mathrm{AD}$ & 3.62 & 5.13 & 1.51 \\
\hline$A D$ dementia & 6.44 & 5.82 & -0.62 \\
\hline Institution, total & 2.87 & 2.35 & -0.51 \\
\hline $\mathrm{MCl}$ due to $\mathrm{AD}$ & 0.06 & 0.09 & 0.03 \\
\hline$A D$ dementia & 2.80 & 2.27 & -0.54 \\
\hline Total LYs (discounted) & 10.45 & 10.70 & 0.26 \\
\hline Total QALYs (discounted) & 5.59 & 6.34 & 0.75 \\
\hline Patient QALYs, total & 6.11 & 6.76 & 0.65 \\
\hline Community, total & 5.27 & 6.06 & 0.80 \\
\hline $\mathrm{MCl}$ due to $\mathrm{AD}$ & 2.65 & 3.63 & 0.98 \\
\hline$A D$ dementia & 2.62 & 2.43 & -0.18 \\
\hline Institution, total & 0.84 & 0.71 & -0.14 \\
\hline $\mathrm{MCl}$ due to $\mathrm{AD}$ & 0.05 & 0.06 & 0.02 \\
\hline AD dementia & 0.80 & 0.64 & -0.16 \\
\hline Lost to ARIA AEs & 0.000 & 0.003 & 0.003 \\
\hline Lost to other AEs & 0.000 & 0.002 & 0.002 \\
\hline Caregiver QALYs lost & 0.52 & 0.42 & -0.09 \\
\hline Total evLYG (discounted) & 5.59 & 6.42 & 0.83 \\
\hline
\end{tabular}

$A D$ Alzheimer's disease, $A d u$ aducanumab, $A E$ adverse event, $A R I A$ amyloid-related imaging abnormalities, evLYG equal value of life-years gained, $L Y$ life-year, $M C I$ mild cognitive impairment, $N A$ not applicable, $N R$ not reached, $Q A L Y$ qualityadjusted life-year, $S O C$ standard of care

transitions to a more severe $\mathrm{AD}$ health state was lower with Adu + SOC than with SOC for all severity levels: to AD dementia $(82.8 \%$ vs. $89.4 \%)$, to moderate $\mathrm{AD}$ dementia or worse (69.5\% vs. $80.0 \%)$, and to severe AD dementia (57.1\% vs. $67.2 \%)$. The model also predicted a lower lifetime probability of transitioning to institutionalization for patients treated with Adu + SOC than for those who received SOC (25.2\% vs. 29.4\%; Table 2; Fig. 2b). Longer median times to transitions to AD dementia and moderate $\mathrm{AD}$ dementia were also predicted for patients in the Adu + SOC group compared with the SOC group (Table 2). Specifically, the median time to $\mathrm{AD}$ dementia (the time when $50 \%$ of those alive had progressed) was estimated to be 1.53 years longer in the Adu + SOC group than in the SOC group (4.30 vs. 2.77 years). The median time to moderate $\mathrm{AD}$ dementia (the time when $50 \%$ of those alive had progressed) was 2.58 years longer for aducanumab-treated patients than for SOC-treated patients ( 7.50 vs. 4.92 years). Of the baseline cohort, aducanumab treatment was estimated to result in an incremental median survival in the MCI due to AD health state of 1.16 years compared with patients who received SOC (3.70 vs. 2.54 years). The model also predicted an 
incremental median survival in the community (of the baseline cohort) of 1.32 years with Adu + SOC compared with SOC (10.17 vs. 8.85 years).

The extrapolation of the clinical benefits observed in EMERGE also translated to potential improvements in time-based outcomes over a lifetime horizon (Table 2). Aducanumab treatment was predicted to increase the expected time per patient in the MCI due to AD health state while reducing the expected time per patient in the $\mathrm{AD}$ dementia health states in both the community and institutionalized settings (Table 2). For patients treated with aducanumab, the incremental change in expected time in MCI due to AD and AD dementia in the community setting was 1.51 and -0.62 years, respectively, compared with patients treated with SOC. The model predicted that the slowing of disease progression would be associated with an indirect survival benefit of 0.38 incremental total undiscounted LYs resulting from treatment with aducanumab compared with patients managed with SOC (13.31 vs. 12.92). Aducanumab treatment was projected to correspond to 0.26 incremental discounted LYs (10.70 vs. 10.45) and 0.75 incremental total QALYs (6.34 vs. 5.59) per patient compared with SOC. Incremental QALYs were estimated at 0.65 for aducanumab-treated patients compared with SOC-managed patients (6.76 vs. 6.11), while incremental caregiver QALYs lost were -0.09 for patients treated with aducanumab compared with SOC, respectively (0.42 vs. 0.52$)$ (Table 2). The QALYs lost due to ARIA and other AEs were estimated to be 0.005 for $\mathrm{Adu}+\mathrm{SOC}$ vs. SOC (Table 2).

\section{Scenario Analyses}

Uncertainty in a model of health outcomes can arise from alternative model settings (population, time horizon, discounting), data sources (selections of natural history transition probabilities), treatment effectiveness assumptions and settings (durability and stopping rules), and structural assumptions (institutionalization and mortality approaches). Therefore, the likely impact of such alternative settings, data sources, and assumptions was assessed via a series of selected scenario analyses that represent plausible variations that may exist in the real world. For the scenario analyses, incremental values for the Adu + SOC versus SOC groups of EMERGE are presented in Table 3, whereas nonincremental values are presented separately for $\mathrm{Adu}+$ SOC and SOC in Table S5 in the electronic supplementary material.

When using time horizons of 10 and 20 years, shorter time horizons did not affect the predicted incremental median transition times to $\mathrm{AD}$ dementia or moderate $\mathrm{AD}$ dementia or incremental median time in the community (Table 3). Furthermore, adjusting the discount rate from $3.0 \%$ in the base case to $0.0 \%$ or $5.0 \%$ in the scenario analyses did not affect incremental median transition time to AD dementia or moderate $\mathrm{AD}$ dementia or median survival in the community (Table 3 ). When older baseline ages (70.0 or 75.0 years) were considered, the incremental median time for transitions to $\mathrm{AD}$ dementia and moderate $\mathrm{AD}$ dementia was increased compared with the base case (65.0 years) (Table 3 ). Increasing the baseline age also lowered the median time of survival in the community compared with the base case (Table 3). In scenario analyses that changed the baseline health state from MCI due to AD to a mixed population of MCI due to $\mathrm{AD}$ and mild $\mathrm{AD}$ dementia (age 70.7 years), a reduction in the incremental median transition time to moderate $\mathrm{AD}$ dementia and median survival in the community was estimated (Table 3).

Scenario analyses were also conducted using alternative data sources for the natural history transition probabilities, for transitions to institutionalization, and for AD-related mortality (Table 3). In the base case, the incremental median times from $\mathrm{MCI}$ due to $\mathrm{AD}$ to $\mathrm{AD}$ dementia and moderate AD dementia were 1.53 and 2.58 years, respectively. In general, most of the scenarios with alternative $\mathrm{AD}$ progression, institutionalization, and mortality data sources showed a limited impact on health outcomes. A scenario analysis using an alternative data source for natural history [55], estimated a slower progression from MCI due to $\mathrm{AD}$ to $\mathrm{AD}$ dementia (13.3\% per year) compared with the base-case analysis (23.2\% per year). The data 


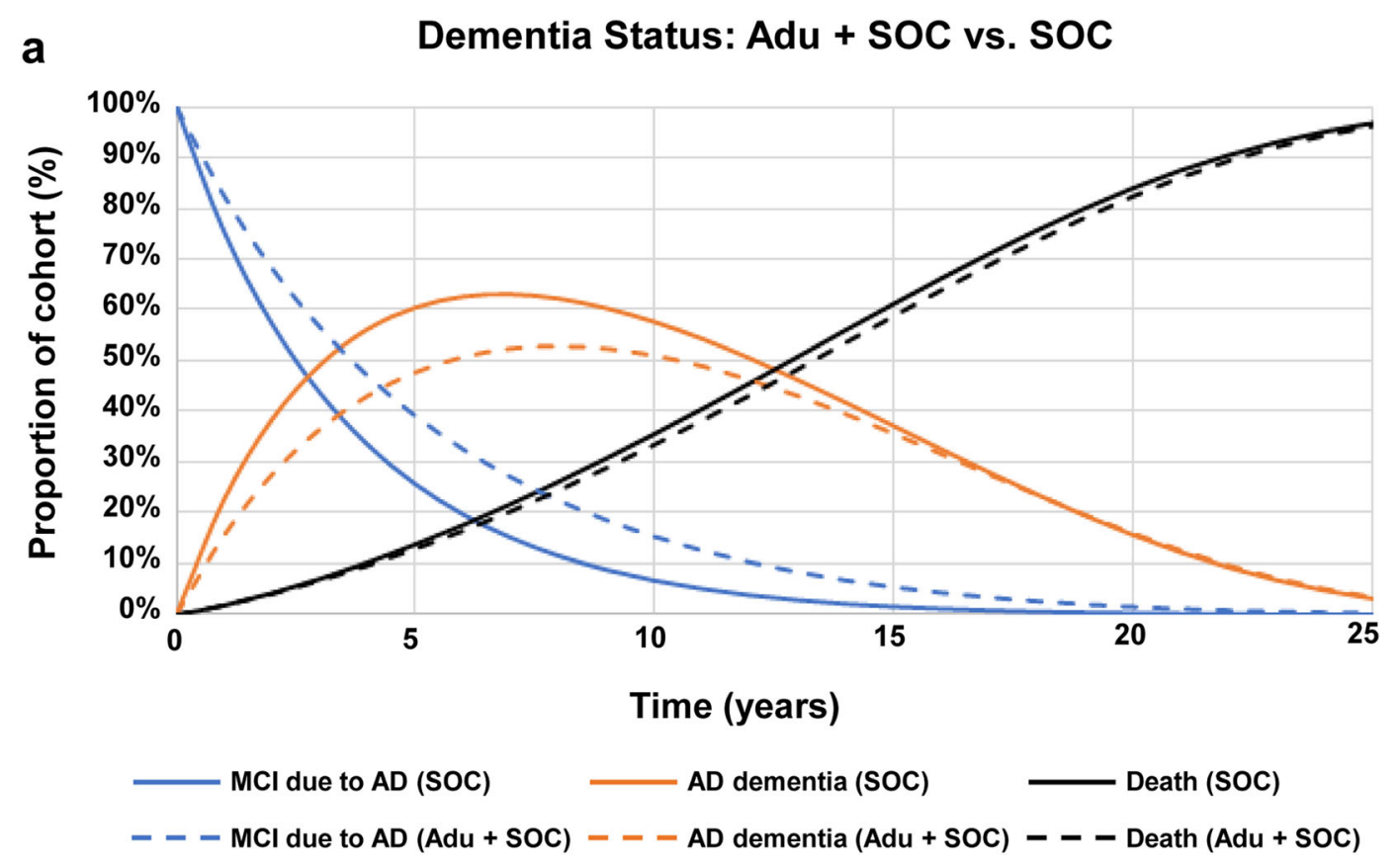

b Care Setting: Adu + SOC vs. SOC

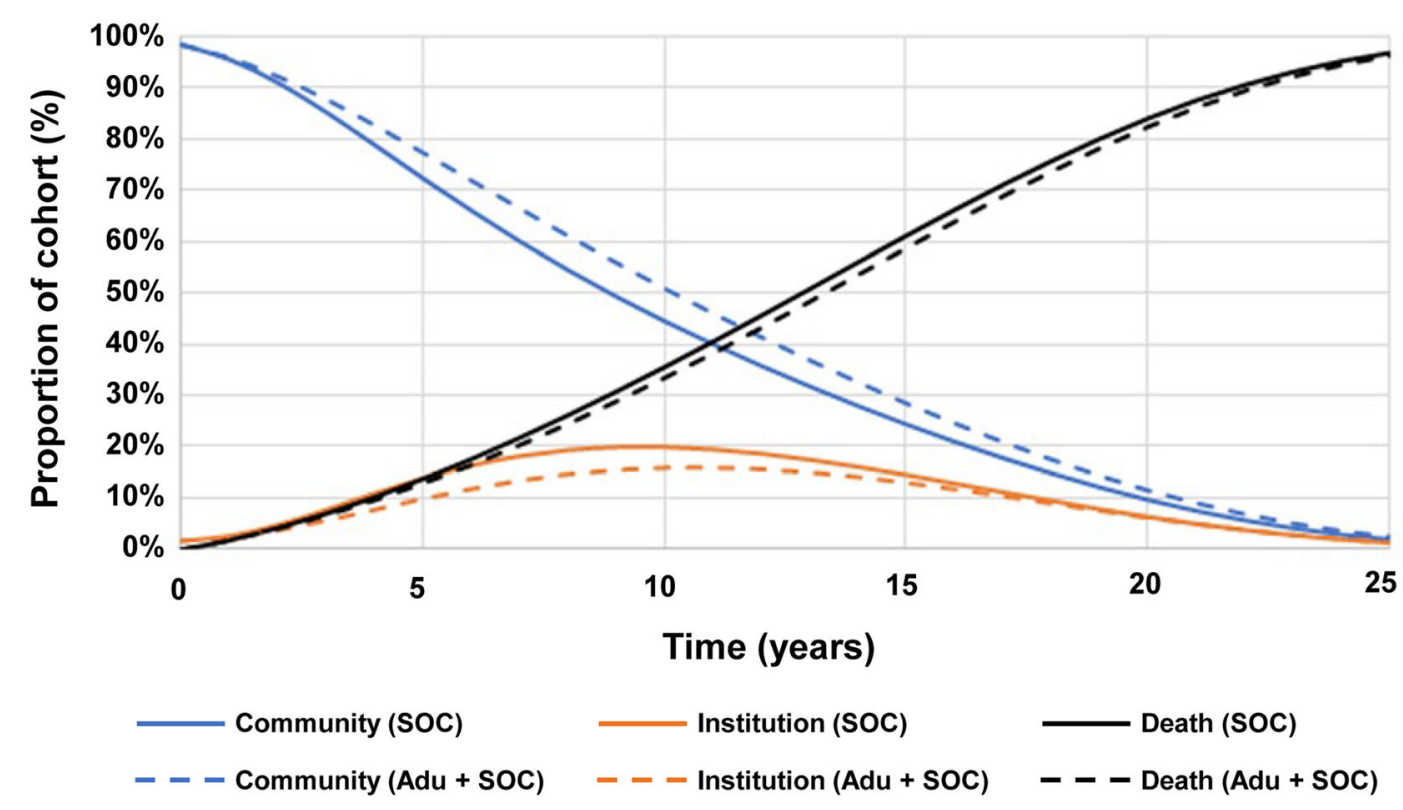

Fig. 2 Markov traces of base-case results. $A D$ Alzheimer's disease, $A d u$ aducanumab, $M C I$ mild cognitive impairment, $S O C$ standard of care

from Iaccarino et al. [55] translated to an increase in incremental median time to $\mathrm{AD}$ dementia ( 3.14 vs. 1.53 years) and moderate $\mathrm{AD}$ dementia (5.32 vs. 2.58 years). A scenario analysis using an alternative data source for progression among mild, moderate, and severe AD health states [30], increased the incremental median time to moderate $\mathrm{AD}$ dementia and reduced the incremental survival in the community, compared with the base case (Table 3). 
Table 3 Incremental health outcomes for selected scenario analyses

\begin{tabular}{|c|c|c|c|c|}
\hline \multirow[b]{2}{*}{ Scenario } & \multicolumn{4}{|c|}{ Incremental outcomes for Adu + SOC vs. SOC } \\
\hline & $\begin{array}{l}\text { Median time to AD } \\
\text { dementia, years }\end{array}$ & $\begin{array}{l}\text { Median time to } \\
\text { moderate } A D \\
\text { dementia, years }\end{array}$ & $\begin{array}{l}\text { Median survival in } \\
\text { community, years }\end{array}$ & QALYs \\
\hline Base-case analysis & 1.53 & 2.58 & 1.32 & 0.75 \\
\hline \multicolumn{5}{|l|}{ Model settings } \\
\hline \multicolumn{5}{|l|}{$\begin{array}{l}\text { Time horizon } \\
\text { base case = lifetime }\end{array}$} \\
\hline 10 years & 1.53 & 2.58 & NA & 0.45 \\
\hline 20 years & 1.53 & 2.58 & 1.32 & 0.72 \\
\hline \multicolumn{5}{|l|}{$\begin{array}{l}\text { Discount rates } \\
\text { base case }=3.0 \% \text { per year }\end{array}$} \\
\hline $0.0 \%$ & 1.53 & 2.58 & 1.32 & 0.98 \\
\hline $5.0 \%$ & 1.53 & 2.58 & 1.32 & 0.63 \\
\hline \multicolumn{5}{|l|}{ Model population } \\
\hline \multicolumn{5}{|l|}{$\begin{array}{l}\text { Baseline age } \\
\text { base case }=65 \text { years }\end{array}$} \\
\hline 70 years & 1.58 & 2.74 & 0.99 & 0.64 \\
\hline 75 years & 1.68 & 3.09 & 0.70 & 0.50 \\
\hline \multicolumn{5}{|l|}{$\begin{array}{l}\text { Baseline health states } \\
\text { base case }=\mathrm{MCl} \text { due to } A D \text { only }\end{array}$} \\
\hline $\begin{array}{l}\text { MCI due to } A D \text { and mild } A D \text { dementia } \\
\text { (Table 1) }\end{array}$ & NA & 2.33 & 0.90 & 0.58 \\
\hline Mild AD dementia only (age $=70.7$ years) & NA & 0.86 & 0.57 & 0.32 \\
\hline \multicolumn{5}{|l|}{ Natural history } \\
\hline \multicolumn{5}{|c|}{$\begin{array}{l}\mathbf{M C l} \text { due to } \mathbf{A D} \text { to } \mathbf{A D} \text { dementia } \\
\text { base case = Potashman et al. } 2020 \text { (35) (23.2\% per } \\
\text { year) }\end{array}$} \\
\hline laccarino et al. 2019 (55) (13.3\% per year) & 3.14 & 5.32 & 1.33 & 0.79 \\
\hline Vos et al. 2015 (56) (26.9\% per year) & 1.32 & 2.76 & 1.37 & 0.77 \\
\hline \multicolumn{5}{|l|}{$\begin{array}{l}\text { Within AD dementia } \\
\text { base case = Potashman et al. } 2020 \text { (35) }\end{array}$} \\
\hline Wimo et al. 2020 (30) data not shown & 1.40 & 3.42 & 0.79 & 0.53 \\
\hline \multicolumn{5}{|l|}{$\begin{array}{l}\text { Institutionalization } \\
\text { base case = Davis et al. } 2018 \text { (36) }\end{array}$} \\
\hline \multicolumn{5}{|l|}{$\begin{array}{l}\text { Mortality } \\
\text { base case = Wilson et al. } 2009(41)\end{array}$} \\
\hline Wimo et al. 2020 (30) data not shown & 1.51 & 2.78 & 1.62 & 0.94 \\
\hline \multicolumn{5}{|l|}{ Aducanumab attributes } \\
\hline \multicolumn{5}{|l|}{$\overline{\text { Efficacy }}$} \\
\hline OTC Cox PH model & 1.88 & 3.16 & 1.55 & 0.88 \\
\hline \multicolumn{5}{|l|}{ Stopping rules } \\
\hline \multicolumn{5}{|l|}{ base case = stop at moderate $A D$ dementia } \\
\hline Maximum of 3 years & 1.05 & 1.02 & 0.51 & 0.33 \\
\hline Maximum of 5 years & 1.53 & 1.72 & 0.81 & 0.48 \\
\hline \multirow{2}{*}{\multicolumn{5}{|c|}{ Discontinuation }} \\
\hline & & & & \\
\hline $7.5 \%$ per yeara & 1.28 & 1.92 & 1.00 & 0.57 \\
\hline
\end{tabular}

$A D$ Alzheimer's disease, $A d u$ aducanumab, $I T T$ intention to treat, $M C I$ mild cognitive impairment, $N A$ not applicable, $O T C$ opportunity to complete, $P H$ proportional hazards, $Q A L Y$ quality-adjusted life-year, SOC standard of care

${ }^{a}$ Discontinuation estimate based on EMERGE trial data

Additional scenarios were conducted to acknowledge the possible impact of treatment stopping rules and discontinuation on the potential long-term clinical benefit of aducanumab (Table 3). Compared with the base case in which aducanumab treatment was stopped at moderate $\mathrm{AD}$ dementia, enforcing a stopping rule of 3 or 5 years shortened the predicted incremental delays in median time to $\mathrm{AD}$ dementia and to moderate $\mathrm{AD}$ dementia and reduced the median survival in the community (Table 3). Discontinuation of aducanumab treatment at a rate of $7.5 \%$ per year, compared with $0 \%$ in the base case, also shortened the 
predicted incremental delays in median time to $\mathrm{AD}$ dementia and to moderate $\mathrm{AD}$ dementia and lowered the median survival in the community.

All scenario analyses were also examined for impacts on predicted incremental QALYs per patient. Compared with the base case, lower QALYs were observed with 10- and 20-year horizons, an increase in age of the population at baseline, and alternative baseline health states of $\mathrm{MCI}$ due to $\mathrm{AD}$ and mild $\mathrm{AD}$ dementia or mild AD dementia only (Table 3). Incremental QALYs increased when the discount rate was lowered to $0.0 \%$. Alternative populations or sources that were used for natural history $[55,56]$ and mortality [30] had different impacts on QALYs. Higher incremental QALYs were estimated in scenario analyses that used alternative sources for progression from MCI due to $\mathrm{AD}$ to $\mathrm{AD}$ dementia $[55,56]$ and mortality [30]. Lower incremental QALYs were predicted with an alternative data source for progression among mild, moderate, and severe AD health states [30] and no change in incremental QALYs was estimated when an alternative source for institutionalization was used [40] (Table 3). Implementation of clinical stopping rules and a treatment discontinuation scenario reduced incremental QALYs compared with the base case (Table 3).

\section{Model Validation}

Model validation was conducted by comparing the predicted outcomes for the SOC with outcomes reported in recently published models for $\mathrm{MCI}$ due to $\mathrm{AD}$ over 10-year and lifetime modeling horizons (Fig. 3 and Table S6 in the electronic supplementary material). The models generally predicted shorter overall survival (i.e., undiscounted total LYs) and less time at each AD severity level as the baseline cohort age increased (ranging from 60 to 80 years), reflecting the role of increasing age-dependent mortality risk across the varying approaches. After baseline cohort age was adjusted for, differences in the model predictions for mean time in state and overall survival could be attributed in most instances to differences in natural history disease progression data (from MCI due to $\mathrm{AD}$ to $\mathrm{AD}$ dementia and within $\mathrm{AD}$ dementia) or mortality data.

The comparison of our model with the SveDem model [30] showed an expected similarity in the time in MCI due to AD owing to comparable amyloid-positive $\mathrm{MCI}$ due to $\mathrm{AD}$ progression data; differences in other time in state predictions and in overall survival reflected the slower overall $\mathrm{AD}$ dementia progression seen in the SveDem analysis and potential differences in the underlying general mortality data between the US and Sweden. While our model and the IPECAD model $[51,52]$ both used data from NACC, our model predicted less time in $\mathrm{MCI}$ due to $\mathrm{AD}$, more time in $\mathrm{AD}$ dementia, and comparable overall survival; these differences were likely attributable to the IPECAD analysis not enforcing an amyloid restriction in their MCI progression estimates. While our model and the Vermunt et al. [53] analysis both considered amyloid-confirmed cohorts, our model consistently predicted less time in all health states. These differences may be partially attributable to the Vermunt et al. [53] analysis allowing transitions from MCI due to $\mathrm{AD}$ to a cognitively normal health state and their reliance on study-observed mortality instead of HRs applied to age-specific general mortality data. Comparisons between our model and the AD-ACE model [54] were feasible only for discounted (3\% annually) total LYs over a 10-year horizon (results not shown in Fig. 3). Our model predicted higher LYs than the AD-ACE analysis, potentially owing to differences between the late MCI cohort from the Alzheimer's Disease Neuroimaging Initiative considered in the ADACE model and the broader, amyloid-confirmed MCI due to AD cohort from NACC considered in our analysis.

\section{DISCUSSION}

The objective of this study was to develop and use an evidence-based modeling approach to predict the potential long-term health outcomes of aducanumab for the treatment of patients with $\mathrm{MCI}$ due to $\mathrm{AD}$ or mild $\mathrm{AD}$ dementia. The analysis presented in this study 
was the first to use efficacy data from a phase 3 clinical trial in a model to estimate possible long-term health outcomes for patients with early AD who were treated with an intervention that reduces $A \beta$ plaques, a biomarker that is reasonably likely to predict clinical benefit $[25,57]$. The population in the current study was restricted to amyloid-confirmed individuals with $\mathrm{AD}$ to align with the EMERGE trial. The high dose of aducanumab used in EMERGE corresponds to the FDA-approved target dose of aducanumab for treatment in real-world clinical practice. This analysis leveraged the best-available published evidence on $\mathrm{AD}$ natural history and quality of life and considered scenarios to reflect the uncertainty of extrapolating clinical trial data to long-term, real-world settings. Thus, this modeling methodology and the predicted health outcomes provide a foundation for healthcare decision-makers and policymakers to understand the potential clinical,

Expected LYs by Disease State (undiscounted, lifetime horizon)

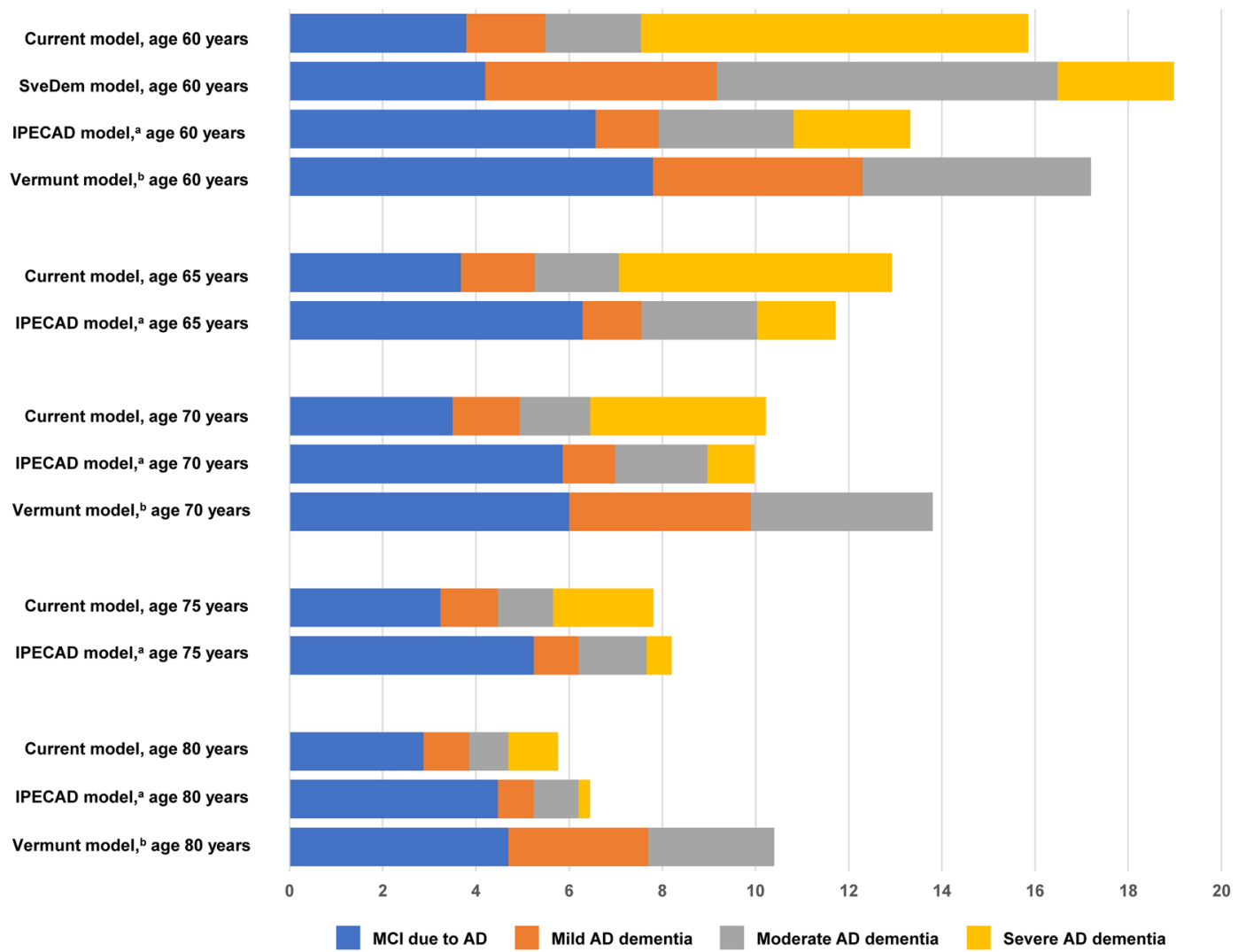

Fig. 3 Comparison with published models of life-years by AD severity level over a lifetime horizon. ${ }^{a}$ The IPECAD model considers up to a 20-year horizon only, which was assumed to approximate a lifetime horizon. For cohorts aged $60,65,70,75$, and 80 years, the IPECAD model predicted that $80.5 \%, 89.1 \%, 94.8 \%, 98.1 \%$, and $99.6 \%$ of patients, respectively, had died at 20 years. ${ }^{\text {b }}$ The multi-state model used in the Vermunt et al. [53] analysis allowed for transitions from $\mathrm{MCI}$ due to $\mathrm{AD}$ back to a preclinical $\mathrm{AD}$ (i.e., no cognitive impairment) health state. For the purposes of reporting, we combined the predicted time in preclinical $\mathrm{AD}$ with the predicted time in $\mathrm{MCI}$ due to AD. Their analysis also combined moderate AD dementia and severe $\mathrm{AD}$ dementia, shown here as moderate $\mathrm{AD}$ dementia. $A D$ Alzheimer's disease, IPECAD International Pharmaco-Economic Collaboration on Alzheimer's Disease, $L Y$ life-year, $M C I$ mild cognitive impairment, SveDem Swedish Dementia registry. Sources: Kansal et al. [54]; Green et al. [51]; Vermunt et al. [53]; Wimo et al. [30]; IPECAD Modeling Group [52] 
economic, and societal value of aducanumab for the treatment of patients with early AD.

The extrapolation of the clinical benefits observed in EMERGE was predicted to translate to improvements in health outcomes over a lifetime horizon. In a cohort of 65-year-old adults with MCI due to $\mathrm{AD}$, treatment with aducanumab was estimated to delay the median time of transition to AD dementia and moderate $\mathrm{AD}$ dementia by 1.53 and 2.58 years, respectively, compared with SOC. The incremental median survival in the $\mathrm{MCI}$ due to $\mathrm{AD}$ health state was estimated at 1.16 for patients treated with aducanumab compared with patients managed with SOC (3.70 vs. 2.54 years). The base-case analysis also predicted 0.75 incremental QALYs per patient for aducanumab compared with the SOC (6.34 vs. 5.59). The scenario analyses around model settings, data sources, and assumptions showed how the magnitude of these clinical benefits varies with alternative baseline cohort characteristics, natural history progression data, aducanumab effectiveness, and settings for stopping treatment with aducanumab. The validation comparisons between the current model and other published models further support the importance of age, natural history progression data, and mortality approach to long-term clinical predictions for patients with MCI due to AD.

Similar potential health outcomes for patients treated with aducanumab in real-world settings would have a profound effect on the informal costs (time spent by caregivers valued in monetary terms) associated with AD. For example, a treatment that could yield a clinically meaningful delay in the onset of $\mathrm{AD}$ has been predicted to result in immediate significant savings in informal costs for patients with $\mathrm{AD}$ and their families [58]. Reducing the number of patients with advancing disease severity as well as the number of LYs spent with dementia is critical to patient and caregiver quality of life [58]. These long-term estimates of the clinical benefits of aducanumab may translate to reduced resource utilization and improved time-based outcomes for patients with $\mathrm{AD}$ and their caregivers.
The estimated median time to AD dementia and to moderate $\mathrm{AD}$ dementia was dependent on the natural history data source. In the base case, the incremental median times from MCI due to $\mathrm{AD}$ to $\mathrm{AD}$ dementia and moderate $\mathrm{AD}$ dementia were 1.53 and 2.58 years, respectively. When an alternative data source with a slower progression from $\mathrm{MCI}$ due to $\mathrm{AD}$ to $\mathrm{AD}$ dementia [55] was used, the incremental median time to $\mathrm{AD}$ dementia and moderate $\mathrm{AD}$ dementia increased. The alternative data source for AD-related mortality [30] increased the incremental median time to moderate $\mathrm{AD}$ dementia and the incremental median survival in the community. These findings highlight the importance of recognizing that incremental outcomes may vary based on underlying $\mathrm{AD}$ progression rates. Discontinuation and stopping rules may also affect the potential clinical benefits of aducanumab in real-world settings. For example, results from the DOMINO clinical trial (NCT00866060) showed that, for patients with moderate or severe $\mathrm{AD}$, continuation of treatment with donepezil resulted in clinically meaningful cognitive benefits [59]. Future analyses should address the importance of maintaining an enduring treatment effect by investigating how stopping or discontinuing aducanumab treatment could affect long-term health outcomes and quality of life.

The analyses presented here are limited by the availability, accuracy, and robustness of the input data and assumptions. Despite the extensive published literature on $\mathrm{AD}$, key uncertainties (e.g., natural history disease progression, especially in patients with confirmed amyloid pathology) and data gaps (e.g., mortality and utility studies spanning the full $\mathrm{AD}$ continuum, caregiver quality of life for institutionalized patients) remain. For example, progression rates along the $\mathrm{AD}$ continuum are highly variable between patients and depend on several factors, such as age, sex, baseline disease stage, cognitive and functional abilities, and levels of $A \beta$ accumulation in the brain [60-62]. Uncertainty in the extrapolation of the treatment effect of aducanumab data from the EMERGE trial is inherent due to the relatively short duration of the trial compared with the lifetime horizon considered in the current 
model. The reliability of the connection between short-term reductions in the CDR-SB rate of decline, as observed in EMERGE, and long-term reductions in $\mathrm{AD}$ progression transition probabilities requires additional follow-up of aducanumab-treated patients. Notably, whether patients treated with aducanumab in the real-world setting will have characteristics and experience levels of treatment efficacy similar to those of EMERGE trial participants is unknown, because $\mathrm{AD}$ is a heterogenous condition in terms of pathophysiological damage and clinical diagnosis.

Studies that will help support the real-world economic impact of aducanumab or other inventions for $\mathrm{AD}$ will be instrumental to performing value assessments now that an intervention that targets underlying $\mathrm{AD}$ pathology has been approved by the FDA. Linking data from national health claims data such as Medicare medical claims through the Research Data Assistance Center program could facilitate the collection of real-world claims data. Medical claims data may also be used to gather health economic data for several years after the end date of a clinical trial [63].

Families of patients with $\mathrm{AD}$, and society at large, shoulder an enormous burden that increases as the neurodegeneration associated with AD worsens and an individual's symptoms progress [17]. The cognitive decline and behavioral disturbances that characterize AD invariably result in functional impairment and the inability to perform daily tasks [9]. In our model, an assumption of one caregiver was used due to a paucity of data outside of patient-caregiver dyads. While a small percentage of patients with dementia (8\%) do not receive help from informal care providers, $30 \%$ of patients with dementia are dependent on three or more unpaid caregivers [3]. Interventions that target the underlying pathology of $\mathrm{AD}$ have the potential to lower this burden and provide immediate as well as longterm value to patients and their families [22].

\section{CONCLUSIONS}

Our analysis predicted that, compared with SOC, treatment with aducanumab would translate to a lower lifetime probability of transitioning to AD dementia, a lower annual probability of transitioning to institutionalization, delays in the median time to transition to AD dementia, and an increased median survival in the community. Longer-term data are needed both through clinical trial follow-up and longitudinal real-world data. The long-term effects of aducanumab on exploratory clinical and biomarker endpoints are being evaluated in the ongoing phase $3 \mathrm{~b}$ Safety and Tolerability study EMBARK (NCT04241068). A future phase 4 confirmatory study will be conducted to further evaluate, verify, and describe the clinical benefit of aducanumab as part of the post-marketing requirements associated with the FDA's accelerated approval pathway under which aducanumab was approved.

\section{ACKNOWLEDGEMENTS}

Funding. This study and the journal's Rapid Service Fee was funded by Biogen (Cambridge, MA, USA).

Medical Writing Support. Medical writing support, under the direction of the authors, was provided by Rosalind Carney, DPhil, of MediTech Media Ltd, and funded by Biogen (Cambridge, MA, USA).

Authorship. All named authors meet the International Committee of Medical Journal Editors (ICMJE) criteria for authorship for this article, take responsibility for the integrity of the work as a whole, and have given their approval for this version to be published.

Authors' Contributions. WLH, IGG, RT, and PP-R conceptualized the study and study design. WLH and IGG developed the model and performed the analyses with input from RT and PP$\mathrm{R}$. FF designed and conducted the post-hoc EMERGE efficacy analysis with input from PP-R. All authors made substantial contributions to the interpretation of the data, critically revised the manuscript for intellectual content, 
approved the final version to be published, and are accountable for all aspects of the work.

Disclosures. William L. Herring is an employee of RTI Health Solutions (RTI-HS), an independent nonprofit research organization. Ian Gopal Gould was an employee of RTI-HS at the time this study was conducted. RTI-HS received funding for this study under a research contract with Biogen International $\mathrm{GmbH}$ (Biogen). Howard Fillit has received fees from Biogen for educational programs, and consulting fees from Samus Therapeutics, Otsuka, and Alector. Peter Lindgren reports advisory boards, speaking assignments and research grants from: Amgen, Astellas, AstraZeneca, BioMarin, European Federation of Pharmaceutical Industries and Associations, Merck Sharp \& Dohme, Novartis, NovoNordisk, and Takeda. Fiona Forrestal, Robin Thompson, and Peter PembertonRoss are employees and shareholders of Biogen.

Compliance with Ethics Guidelines. The EMERGE trial was conducted in accordance with Declaration of Helsinki and the International Conference on Harmonization and Good Clinical Practice guidelines and was approved by ethics committees or institutional review boards at each site. All patients provided written informed consent. An independent data monitoring committee was responsible for monitoring the conduct of the trials, assessing safety routinely, and reviewing futility analysis results. This article is based on previously conducted studies and does not contain any new studies with human participants or animals performed by any of the authors.

Data Availability. All data used for this study are provided in the manuscript. Additional details are available from the corresponding author on request.

Open Access. This article is licensed under a Creative Commons Attribution-NonCommercial 4.0 International License, which permits any non-commercial use, sharing, adaptation, distribution and reproduction in any medium or format, as long as you give appropriate credit to the original author(s) and the source, provide a link to the Creative Commons licence, and indicate if changes were made. The images or other third party material in this article are included in the article's Creative Commons licence, unless indicated otherwise in a credit line to the material. If material is not included in the article's Creative Commons licence and your intended use is not permitted by statutory regulation or exceeds the permitted use, you will need to obtain permission directly from the copyright holder. To view a copy of this licence, visit http://creativecommons.org/licenses/by$\mathrm{nc} / 4.0 /$.

\section{REFERENCES}

1. Alzheimer's Society UK. Facts for the media 2021. https://www.alzheimers.org.uk/about-us/news-andmedia/facts-media.

2. World Health Organization. Dementia: number of people affected to triple in next 30 years 2017. https://www.who.int/news/item/07-12-2017dementia-number-of-people-affected-to-triple-innext-30-years. Accessed 27 July 2021.

3. Alzheimer's Association. 2020 Alzheimer's disease facts and figures. https://alz-journals.onlinelibrary. wiley.com/doi/full/10.1002/alz.12068. Accessed 27 July 2021.

4. Centers for Disease Control and Prevention. Caregiving for a person with Alzheimer's disease or a related dementia. https://www.cdc.gov/aging/ caregiving/alzheimer.htm. Accessed 27 July 2021.

5. Hardy JA, Higgins GA. Alzheimer's disease: the amyloid cascade hypothesis. Science. 1992;256(5054):184-5.

6. Dubois B, Hampel H, Feldman HH, Scheltens P, Aisen P, Andrieu S, et al. Preclinical Alzheimer's disease: definition, natural history, and diagnostic criteria. Alzheimers Dement. 2016;12(3):292-323.

7. Price JL, Morris JC. Tangles and plaques in nondemented aging and "preclinical" Alzheimer's disease. Ann Neurol. 1999;45(3):358-68.

8. Sperling RA, Aisen PS, Beckett LA, Bennett DA, Craft $S$, Fagan AM, et al. Toward defining the preclinical stages of Alzheimer's disease: recommendations from the National Institute on Aging-Alzheimer's Association workgroups on diagnostic guidelines 
for Alzheimer's disease. Alzheimers Dement. 2011;7(3):280-92.

9. Jack CR Jr, Bennett DA, Blennow K, Carrillo MC, Dunn B, Haeberlein SB, et al. NIA-AA Research Framework: toward a biological definition of Alzheimer's disease. Alzheimers Dement. 2018;14(4): 535-62.

10. Centers for Disease Control and Prevention. CDC Wonder. Accessed [Jul 27, 2021]. https://wonder. cdc.gov/

11. Knopman DS, Gottesman RF, Sharrett AR, Wruck LM, Windham BG, Coker L, et al. Mild cognitive impairment and dementia prevalence: the Atherosclerosis Risk in Communities Neurocognitive Study (ARIC-NCS). Alzheimers Dement. 2016;2:1-11.

12. Lopez-Anton R, Santabarbara J, De-la-Camara C, Gracia-Garcia P, Lobo E, Marcos G, et al. Mild cognitive impairment diagnosed with the new DSM-5 criteria: prevalence and associations with non-cognitive psychopathology. Acta Psychiatr Scand. 2015;131(1):29-39.

13. Petersen RC, Lopez O, Armstrong MJ, Getchius TSD, Ganguli M, Gloss D, et al. Practice guideline update summary: mild cognitive impairment: report of the Guideline Development, Dissemination, and Implementation Subcommittee of the American Academy of Neurology. Neurology. 2018;90(3): 126-35.

14. Rabinovici GD, Gatsonis C, Apgar C, Chaudhary K, Gareen I, Hanna L, et al. Association of amyloid positron emission tomography with subsequent change in clinical management among medicare beneficiaries with mild cognitive impairment or dementia. JAMA. 2019;321(13):1286-94.

15. Robinson RL, Rentz DM, Andrews JS, Zagar A, Kim $\mathrm{Y}$, Bruemmer V, et al. Costs of early stage Alzheimer's disease in the United States: cross-sectional analysis of a prospective cohort study (GERAS-US) 1 . J Alzheimers Dis. 2020;75(2):437-50.

16. Wimo A, Reed CC, Dodel R, Belger M, Jones RW, Happich M, et al. The GERAS Study: a prospective observational study of costs and resource use in community dwellers with Alzheimer's disease in three European countries-study design and baseline findings. J Alzheimers Dis. 2013;36(2):385-99.

17. El-Hayek YH, Wiley RE, Khoury CP, Daya RP, Ballard C, Evans AR, et al. Tip of the Iceberg: assessing the global socioeconomic costs of Alzheimer's disease and related dementias and strategic implications for stakeholders. J Alzheimers Dis. 2019;70(2): 323-41.
18. Gustavsson A, Brinck P, Bergvall N, Kolasa K, Wimo A, Winblad B, et al. Predictors of costs of care in Alzheimer's disease: a multinational sample of 1222 patients. Alzheimers Dement. 2011;7(3):318-27.

19. Winblad B, Amouyel P, Andrieu S, Ballard C, Brayne C, Brodaty H, et al. Defeating Alzheimer's disease and other dementias: a priority for European science and society. Lancet Neurol. 2016;15(5): $455-532$.

20. Deb A, Thornton JD, Sambamoorthi U, Innes K. Direct and indirect cost of managing Alzheimer's disease and related dementias in the United States. Expert Rev Pharmacoecon Outcomes Res. 2017;17(2):189-202.

21. Gustavsson A, Pemberton-Ross P, Gomez Montero M, Hashim M, Thompson R. Challenges in demonstrating the value of disease-modifying therapies for Alzheimer's disease. Expert Rev Pharmacoecon Outcomes Res. 2020;20(6):563-70.

22. Makin C, Neumann P, Peschin S, Goldman D. Modelling the value of innovative treatments for Alzheimer's disease in the United States. J Med Econ. 2021;24(1):764-9.

23. Cummings JL, Tong G, Ballard C. Treatment combinations for Alzheimer's disease: current and future pharmacotherapy options. J Alzheimers Dis. 2019;67(3):779-94.

24. Arvanitakis Z, Shah RC, Bennett DA. Diagnosis and management of dementia: review. JAMA. 2019;322(16):1589-99.

25. Aduhelm (aducanumab-avwa). Prescribing information. Biogen, Inc; 2021 Accessed [July 27, 2021]. https://www.accessdata.fda.gov/drugsatfda_docs/ label/2021/761178s000lbl.pdf

26. Sevigny J, Chiao P, Bussiere T, Weinreb PH, Williams $\mathrm{L}$, Maier $\mathrm{M}$, et al. The antibody aducanumab reduces $A \beta$ plaques in Alzheimer's disease. Nature. 2016;537(7618):50-6.

27. Budd D, Burns LC, Guo Z, L'Italien G, Lapuerta P. Impact of early intervention and disease modification in patients with predementia Alzheimer's disease: a Markov model simulation. Clinicoecon Outcomes Res. 2011;3:189-95.

28. Djalalov S, Yong J, Beca J, Black S, Saposnik G, Musa $Z$, et al. Genetic testing in combination with preventive donepezil treatment for patients with amnestic mild cognitive impairment: an exploratory economic evaluation of personalized medicine. Mol Diagn Ther. 2012;16(6):389-99.

29. Skoldunger A, Johnell K, Winblad B, Wimo A. Mortality and treatment costs have a great impact 
on the cost-effectiveness of disease modifying treatment in Alzheimer's disease-a simulation study. Curr Alzheimer Res. 2013;10(2):207-16.

30. Wimo A, Handels R, Winblad B, Black CM, Johansson G, Salomonsson S, et al. Quantifying and describing the natural history and costs of Alzheimer's disease and effects of hypothetical interventions. J Alzheimers Dis. 2020;75(3):891-902.

31. O'Bryant SE, Waring SC, Cullum CM, Hall J, Lacritz L, Massman PJ, et al. Staging dementia using Clinical Dementia Rating Scale Sum of Boxes scores: a Texas Alzheimer's research consortium study. Arch Neurol. 2008;65(8):1091-5.

32. Siebert U, Alagoz O, Bayoumi AM, Jahn B, Owens DK, Cohen DJ, et al. State-transition modeling: a report of the ISPOR-SMDM modeling good research practices task force-3. Value Health. 2012;15(6): 812-20.

33. Albert MS, DeKosky ST, Dickson D, Dubois B, Feldman HH, Fox NC, et al. The diagnosis of mild cognitive impairment due to Alzheimer's disease: recommendations from the National Institute on Aging-Alzheimer's Association workgroups on diagnostic guidelines for Alzheimer's disease. Alzheimers Dement. 2011;7(3):270-9.

34. McKhann GM, Knopman DS, Chertkow H, Hyman BT, Jack CR Jr, Kawas $\mathrm{CH}$, et al. The diagnosis of dementia due to Alzheimer's disease: recommendations from the National Institute on Aging-Alzheimer's Association workgroups on diagnostic guidelines for Alzheimer's disease. Alzheimers Dement. 2011;7(3):263-9.

35. Potashman M, Buessing M, Benea ML, Cummings J, Borson S, Pemberton-Ross P, et al., editors. Estimating progression rates across the spectrum of Alzheimer's disease for amyloid positive individuals using National Alzheimer's Coordinating Center data. Clinical Trials on Alzheimer's Disease Congress 2020; 2020. https://www.ncbi.nlm.nih.gov/ pmc/articles/PMC7772113/ shown as J Prev Alzheimers Dis. 202;7(suppl 1):55-119. Poster 070. Accessed 27 July 2021.

36. Davis M, Thomas OC, Johnson S, Cline S, Merikle E, Martenyi $\mathrm{F}$, et al. Estimating Alzheimer's disease progression rates from normal cognition through mild cognitive impairment and stages of dementia. Curr Alzheimer Res. 2018;15(8):777-88.

37. Hauber AB, Gnanasakthy A, Snyder EH, Bala MV, Richter A, Mauskopf JA. Potential savings in the cost of caring for Alzheimer's disease. Treatment with rivastigmine. Pharmacoeconomics. 2000;17(4):351-60.
38. Neumann PJ, Hermann RC, Kuntz KM, Araki SS, Duff SB, Leon J, et al. Cost-effectiveness of donepezil in the treatment of mild or moderate Alzheimer's disease. Neurology. 1999;52(6):1138-45.

39. Spackman DE, Kadiyala S, Neumann PJ, Veenstra DL, Sullivan SD. Measuring Alzheimer disease progression with transition probabilities: estimates from NACC-UDS. Curr Alzheimer Res. 2012;9(9): 1050-8.

40. Neumann PJ, Kuntz KM, Leon J, Araki SS, Hermann RC, Hsu MA, et al. Health utilities in Alzheimer's disease: a cross-sectional study of patients and caregivers. Med Care. 1999;37(1):27-32.

41. Wilson RS, Aggarwal NT, Barnes LL, Bienias JL, Mendes de Leon CF, Evans DA. Biracial population study of mortality in mild cognitive impairment and Alzheimer disease. Arch Neurol. 2009;66(6): 767-72.

42. Arias E, Xu J. United States life tables, 2017. Natl Vital Stat Rep. 2019;68(7):1-66.

43. Andersen K, Lolk A, Martinussen T, Kragh-Sorensen P. Very mild to severe dementia and mortality: a 14-year follow-up-the Odense study. Dement Geriatr Cogn Disord. 2010;29(1):61-7.

44. von Rosenstiel P, Haeberlein S, Castrillo-Viguera C, Chen T, O'Gorman J, Rajagovindan R, et al., editors. Aducanumab 48-month analyses from PRIME, a phase $1 \mathrm{~b}$ study in patients with early Alzheimer's disease. 11th Clinical Trials on Alzheimer's Disease Congress 2018; 2018; Barcelona, Spain.

45. Landeiro F, Mughal S, Walsh K, Nye E, Morton J, Williams $\mathrm{H}$, et al. Health-related quality of life in people with predementia Alzheimer's disease, mild cognitive impairment or dementia measured with preference-based instruments: a systematic literature review. Alzheimers Res Ther. 2020;12(1):154.

46. Hongisto K, Vaatainen S, Martikainen J, Hallikainen I, Valimaki T, Hartikainen S, et al. Self-rated and caregiver-rated quality of life in Alzheimer disease with a focus on evolving patient ability to respond to questionnaires: 5-year prospective ALSOVA Cohort Study. Am J Geriatr Psychiatry. 2015;23(12): 1280-9.

47. Mesterton J, Wimo A, By A, Langworth S, Winblad B, Jonsson L. Cross sectional observational study on the societal costs of Alzheimer's disease. Curr Alzheimer Res. 2010;7(4):358-67.

48. Janssen B, Szende A. Population norms for the EQ5D. In: Szende A, Janssen B, Cabases J, editors. Selfreported population health: an international perspective based on EQ-5D. Dordrecht: Springer; 2014. p. 19-30. 
49. ICER. A guide to ICER's methods for health technology assessment. 2018. http://icer-review.org/ wp-content/uploads/2018/08/ICER-HTA-Guide_ 082018.pdf. Accessed 27 July 2021.

50. Sanders GD, Neumann PJ, Basu A, Brock DW, Feeny D, Krahn M, et al. Recommendations for conduct, methodological practices, and reporting of cost-effectiveness analyses: second panel on cost-effectiveness in health and medicine. JAMA. 2016;316(10):1093-103.

51. Green C, Handels R, Gustavsson A, Wimo A, Winblad B, Skoldunger A, et al. Assessing cost-effectiveness of early intervention in Alzheimer's disease: An open-source modeling framework. Alzheimers Dement. 2019;15(10):1309-21.

52. IPECAD Modelling Group. The IPECAD OpenSource Model Version 1.1. 20212021 [Available from: https://ipecadgroup.wordpress.com/opensource-model/. Accessed 1 Mar 2021.

53. Vermunt L, Sikkes SAM, van den Hout A, Handels R, Bos I, van der Flier WM, et al. Duration of preclinical, prodromal, and dementia stages of Alzheimer's disease in relation to age, sex, and APOE genotype. Alzheimers Dement. 2019;15(7):888-98.

54. Kansal AR, Tafazzoli A, Ishak KJ, Krotneva S. Alzheimer's disease archimedes condition-event simulator: development and validation. Alzheimers Dement (NY). 2018;4:76-88.

55. Iaccarino L, Sala A, Perani D. Alzheimer's disease neuroimaging I. Predicting long-term clinical stability in amyloid-positive subjects by FDG-PET. Ann Clin Transl Neurol. 2019;6(6):1113-20.

56. Vos SJ, Verhey F, Frolich L, Kornhuber J, Wiltfang J, Maier W, et al. Prevalence and prognosis of
Alzheimer's disease at the mild cognitive impairment stage. Brain. 2015;138(Pt 5):1327-38.

57. US Food and Drug Administration. Expedited programs for serious conditions-drugs and biologics. 2014. https://www.fda.gov/regulatory-information/ search-fda-guidance-documents/expeditedprograms-serious-conditions-drugs-and-biologics. Accessed 27 July 2021.

58. Alzheimer's Association. Changing the trajectory of Alzheimer's disease: How a treatment by 2025 saves lives and dollars 2015. https://www.alz.org/media/ documents/changing-the-trajectory-r.pdf. Accessed 27 July 2021.

59. Howard R, McShane R, Lindesay J, Ritchie C, Baldwin A, Barber R, et al. Donepezil and memantine for moderate-to-severe Alzheimer's disease. N Engl J Med. 2012;366(10):893-903.

60. Harrington KD, Lim YY, Ames D, Hassenstab J, Laws SM, Martins RN, et al. Amyloid beta-associated cognitive decline in the absence of clinical disease progression and systemic illness. Alzheimers Dement (Amst). 2017;8:156-64.

61. Ito K, Ahadieh S, Corrigan B, French J, Fullerton T, Tensfeldt $\mathrm{T}$, et al. Disease progression meta-analysis model in Alzheimer's disease. Alzheimers Dement. 2010;6(1):39-53.

62. Wattmo C, Wallin AK, Minthon L. Progression of mild Alzheimer's disease: knowledge and prediction models required for future treatment strategies. Alzheimers Res Ther. 2013;5(5):44.

63. Caro J, Klittich W, McGuire A, Ford I, Norrie J, Pettitt D, et al. The West of Scotland coronary prevention study: economic benefit analysis of primary prevention with pravastatin. BMJ. 1997;315(7122):1577-82. 